\title{
Assessment of hospital environments and policies: seeking an age-friendly hospital
}

\section{Abstract}

Functional decline is one of the main problems arising from the hospitalization of elderly persons. Hospital environments and policies focused on functionality are crucial for diminishing disabilities associated with hospitalization. This observational study was performed in four internal medicine services and aimed to evaluate the hospital environment and policies as an integral step in implementing the Function-Focused Care (FFC) programme being established in Portugal. The results of the hospital environment analysis suggest that design and product support, while present, are limited given the needs, complexities and specificities of hospitalized elderly persons. Policy evaluation indicated the existence of institutional projects that reflect a concern with functionality. However, more than half of the criteria evaluated need to be developed further in order to integrate the FFC programme. These results highlight that, while these services aim to be more "age-friendly environments", a number of initiatives can and should be undertaken in order to guide the FFC implementation process. This will benefit patients and their families, professionals and institutions.

Keywords: Elderly. Hospitals. Environment. Policy.

\footnotetext{
Centro Hospitalar e Universitário de Coimbra, Serviço de Urgência. Coimbra, Portugal.

Centro Hospitalar e Universitário de Coimbra, Cirurgia-Máxilo Facil e Plástica. Coimbra, Portugal.

Centro Hospitalar e Universitário de Coimbra, Psiquiatria Mulheres. Coimbra, Portugal. 


\section{INTRODUCTION}

Data from the last Portuguese census (2012) indicate that $19 \%$ of the population is 65 years or older $^{1}$. This group has the highest hospital admission rates of all age ranges ${ }^{2}$. The negative impact of hospitalization has been described in literature, and is entitled "hospitalization associated disability (HAD)" ${ }^{3}$. At least $30 \%$ of people aged 70 years or older are discharged with disability in an activity of daily living that they did not have prior to hospitalization ${ }^{4}$. The HAD model is considered a geriatric syndrome, with concentric and interactive risk factors. This syndrome can occur before (onset of acute illness), during and after hospitalization. The following risk factors have been identified: reduced functional reserve due to acute disease (old age, decline in physical function, decline in cognitive function, geriatric syndromes, depression and lack of social support ${ }^{3,5,6}$, the severity of the acute disease; hospitalization factors (a stressful physical environment which is not adapted for the elderly, restriction of mobility, malnutrition, forced dependence); and post-hospitalization factors (quality of discharge planning, housing conditions, economic and social resources, and community support). Based on the intrinsic and extrinsic etiology of the risk factors for HAD, Resnick et al. ${ }^{7}$ developed Function Focused Care (FFC) which aims to promote and achieve the highest degree of functionality and prevent physical and cognitive deconditioning. FFC is based on the Social-Ecological Model that frames the interrelationship between intrapersonal, interpersonal, environmental and political factors, which may contribute to the impact of hospitalization on the elderly ${ }^{8}$. The use of this model is also fundamental in guiding the implementation process of FFC to benefit these users ${ }^{8}$. This process includes four steps: a) assessment of policies and environment; b) education; c) setting goals/objectives for FFC; and d) motivation and mentoring. The study "Promotion of FFC of Hospitalized Elderly Persons" was carried out in four internal medicine services in an academic hospital in the central region of Portugal. A quasiexperimental study was carried out, with two wards in the case group (normal care) and two in the control group (implementation of the FFC program). Stage A of the implementation is based on the framework of the "age-friendly hospital" which seeks to promote safety, minimize functional decline and mitigate adverse medical and social outcomes for elderly persons?. As such, this brief communication aims to report the results of this first phase of the study on FFC.

\section{METHOD}

A descriptive observational study was developed in four internal medicine units of a hospital located in the central region of Portugal. Initially, the study was presented to the service director of the internal medicine units and to the respective head nurses, and the visit to the services was formalized (March 2016).

The evaluation of the policies and environments that promoted functionality and physical activity was based on the checklists developed by Resnick et al. ${ }^{8}$. These were translated and adapted by the researchers (JT, LG and LN). The service/institution policy checklist includes 15 items that evaluate policies/ protocols that optimize functionality and physical activity (e.g., physical restraints, constraints, and walking). The environmental checklist includes 18 items that evaluate the environmental factors that promote or inhibit FFC (e.g. unobstructed walking areas, suitable lighting of spaces, height of chairs, beds and toilets). For each of the items of these checklists the presence (1 point) or absence (zero points) of measures favoring FFC is indicated. The sum of the answers is then calculated. The higher the scores, the more the environment and the policies of these services are "age-friendly" and promoters of functionality.

Data collection was performed through an open interview with the head nurses/heads of the services and a guided tour of the units by the three investigators (external). These had an average duration of 1 hour and 30 minutes and were based on the checklists. We attempted to minimize bias by familiarizing the researchers with the items on the checklists, and specific characteristics were defined for each of the items. Each researcher individually completed the checklists. The responses were then analyzed, and $100 \%$ agreement was obtained for all items. 
The present study was approved by the Ethics Commission of the Hospital, under approval $\mathrm{N}^{\circ}$ CHUC-065-14.

\section{RESULT}

The results of the evaluation of environments and policies that promote FFC are shown in Table 1. The environmental scores varied between five and nine points, with a mean of $6.8 \pm 1.7$. This contrasted with the policies/protocols scores, which had a mean of $7 \pm 0$, with each unit scoring seven. In general, the heights of the beds, chairs and toilets were adequate, night lights were provided, and there were pleasant common areas (lounges), although there were occasional differences between services. Support products are scarce and focused on wheelchairs, walkers, crutches, transfer lifts, electric folding beds with trapezes for mobilization and wheelchair work desks. The footwear (bedroom slippers) made available by the institution did not make walking easy, and support tables did not facilitate eating out of bed. It was found that the areas of the units are managed according to space and the need to hold equipment. Due to space constraints, free areas that promote functionality (walking, gait training) are limited and unsafe.

Analysis of the policies/protocols indicated the existence of institutional projects, such as the prevention of pressure ulcers, performance evaluation and a computerized nursing documentation system, reflecting a concern for functionality. However, more than half of the evaluation items (e.g., physical restraints, constraints, Foley catheter, and walking, among others) require development if FFC is to be integrated.

Table 1. Outcomes of the evaluation of the environment and policies promoting Function-Focused Care in four internal medicine services in a central hospital. Coimbra, Portugal, 2016.

\begin{tabular}{lll}
\hline Service & $\begin{array}{l}\text { Environment (18 ítems) } \\
\mathrm{n}(\%)\end{array}$ & $\begin{array}{l}\text { Policies (15 ítems) } \\
\mathrm{n}(\%)\end{array}$ \\
\hline 1 & $7(38.9 \%)$ & $7(46.7 \%)$ \\
\hline 2 & $9(50 \%)$ & $7(46.7 \%)$ \\
\hline 3 & $5(27.8 \%)$ & $7(46.7 \%)$ \\
\hline
\end{tabular}

\section{DISCUSSION}

This evaluation is part of the first step of implementing a program to promote functionality ${ }^{7}$, based on the assumptions of the Social-Ecological Model. In this sense, the model is not limited to the development of care, but also analyzes how environment (services) and policies (institution) should be mobilized and oriented in order to promote functionality. The results indicate that the hospital environment and policies should focus on promoting independence in order to prevent and/ or mitigate HAD. The hospital environment has an impact on the mobility of the elderly ${ }^{10}$. When this environment is unfamiliar, with technical equipment, frequent jargon and the disruption of routines and habits, it becomes a stress generator for such individuals, challenging their adaptive capacities and preventing their recovery ${ }^{9}$. It is important to consider that the hospital under analysis was designed in the 1950s and built in the 1980s, when demographic, epidemiological and social transition had not taken place in Portugal. As such, the indoor and outdoor areas do not reflect the current concerns of "age-friendly" hospitals. Additionally, the shortage of support products, notably food, clothing, hygiene and walking related, can make it difficult to practice $\mathrm{FFC}^{7}$. 
Hospitals with gerontic-geriatric sensibility, based in the physical design and policies/protocols, can compensate for the decline of the capacities of this population. A functionality-centric view can protect this age group, maximize its independence, and ameliorate the changes associated with primary aging, compensating for cognitive, sensory, and physical decline.

The greater variability in the environment scores may be the result of the existing resources, the management of the spaces and the focus that is given to the physical activity and promotion of independence of hospitalized elderly persons. The homogeneity in the policies/protocols scores stems from the fact that projects are institutional, reinforcing the idea that the more guidelines that seek to reflect the functionality or the prevention of HAD, the greater the impact on the elderly.

This evaluation was limited to a visit to four services, and generalization to other units in the same institution was impossible. A longer evaluation period could allow more profound analysis of some of the items studied, and permit observance of the interaction between the environment and the development of care. The checklists used focus on general aspects, and therefore other, more specific instruments with a multidimensional perspective should be considered ${ }^{8}$. Finally, this study only considered the internal areas

\section{REFERENCES}

1. Instituto Nacional de Estatística. Censos 2011. Lisboa: INE; 2012.

2. Direção Geral da Saúde. Centros de Saúde e Unidades Hospitalares: Recursos e produção do SNS: ano de 2008. Lisboa: Direcção-Geral da Saúde; 2009. p. 122.

3. Covinsky KE, Pierluissi E, Story THEPS. Hospitalization-Associated Disability "She Was Probably Able to Ambulate, but I 'm Not Sure." J Am Med Assoc. 2011;306(16):1782-93. of the hospital, while an adjusted environment should also include external areas.

\section{CONCLUSION}

The results of the present study emphasize that although these units are moving towards being more "age-friendly", a number of initiatives can and should be undertaken so that hospitalization does not represent a loss of functionality. It was demonstrated that, although some good institutional practices were in place, it is important to incorporate other protocols/procedures that focus on walking, spaces that promote functionality, and physical restraints, among other policies. The focus on the prevention of complications resulting from the hospitalization of the elderly should direct the hospital administration, since it is possible to improve care for the patient/ family, the professionals and the institution. This study, under the aegis of the Nursing Directorate, denotes increasing concern regarding this issue among the organization.

\section{ACKNOWLEDGEMENTS}

The author would like to thank the Director of Nursing of the Centro Hospitalar e Universitário de Coimbra (Coimbra Hospital and University Center) (CHUC), António Marques.

4. Covinsky KE, Palmer RM, Fortinsky RH, Counsell SR, Stewart AL, Kresevic D, et al. Loss of independence in activities of daily living in older adults hospitalized with medical illness: Increased vulnerability with age. J Am Geriatr Soc. 2003;51:451-8.

5. Da Cunha FCM, Cintra MTG, Da Cunha LCM, Giacomin KC, Couto EAB. Fatores que predispõem ao declínio funcional em idosos hospitalizados . Rev Bras Geriatr Gerontol. 2009;12(3):475-87. 
6. Hoogerduijn JG, Schuurmans MJ, Korevaar JC, Buurman BM, De Rooij SE. Identification of older hospitalised patients at risk for functional decline, a study to compare the predictive values of three screening instruments. J Clin Nurs. 2010;19(910):1219-25.

7. Res J Clin Nurs.nick B, Boltz M, Galik E, PretzerAboff I. Restorative care nursing for older adults. New York: Springer Publishing Company; 2012.
8. Boltz M, Resnick B, Capezuti E, Shuluk J, Secic M. Functional decline in hospitalized older adults: can nursing make a difference? Geriatr Nurs. 2012;33(4):272-9.

9. Parke B, Friesen K. Code Plus: Physical design components for an elder friendly hospital. 2nd ed. Fraser H, Parke B, Friesen K, editors. Alberta: Fraser Health; 2015.

10. Zisberg A, Syn-Hershko A. Factors related to the mobility of hospitalized older adults: a prospective cohort study. Geriatr Nurs. 2016;37(2):96-100.

Received: June 16, 2016

Reviewed: January 05, 2017

Accepted: March 28, 2017 